\title{
Wave diffraction by a cosmic string
}

\author{
Isabel Fernández-Núñez ${ }^{\mathrm{a}, \mathrm{b}}$, Oleg Bulashenko ${ }^{\mathrm{a}}$ \\ ${ }^{a}$ Departament de Física Quàntica i Astrofísica \\ ${ }^{b}$ Institut de Ciències del Cosmos (ICCUB) \\ Facultat de Física, Universitat de Barcelona, Martí i Franquès 1, E-08028 Barcelona, Spain.
}

\begin{abstract}
We show that if a cosmic string exists, it may be identified through characteristic diffraction pattern in the energy spectrum of the observed signal. In particular, if the string is on the line of sight, the wave field is shown to fit the Cornu spiral. We suggest a simple procedure, based on Keller's geometrical theory of diffraction, which allows to explain wave effects in conical spacetime of a cosmic string in terms of interference of four characteristic rays. Our results are supposed to be valid for scalar massless waves, including gravitational waves, electromagnetic waves, or even sound in case of condensed matter systems with analogous topological defects.
\end{abstract}

Keywords: Cosmic Strings, Topological defects, Gravitational lensing, Diffraction

\section{Introduction}

Topological defects may appear naturally during a symmetry-breaking phase transition in various physical systems. One of the examples is a cosmic string a long-lived topologically stable structure that may have been formed at phase transitions in the early Universe [1-3]. Cosmic strings are analogous to other linear defects found in condensed matter systems: vortex lines in liquid helium [4], flux tubes in type-II superconductors [5], disclinations in liquid crystals [6], in graphene [7], or in metamaterials [8-10].

The spacetime around a straight cosmic string is locally flat, but it globally has a conical topology that can give rise to a variety of observable phenomena $[2,3]$. The most evident way to detect cosmic strings is by means of gravitational lensing. The conical topology should produce double images of a distant source situated behind the string [11]. The images should be undistorted but they may overlap if the split angle, which is proportional to the string tension, is small. In such a case, the wave effects are extremely important as a probe in gravitational lensing [12], that was extensively studied for compact or point-like objects [13], but only a few studies are known for the strings [14-18].

In this Letter we show that the wave propagation in conical spacetime, caused by a cosmic string or similar topological defects, can be effectively treated in the framework of the celebrated Arnold Sommerfeld's half-plane diffraction 
problem [19-21]. In this way, we find analytical solutions in terms of Fresnel integrals, that let us to conclude that the wave effects in conical space are determined by a unique parameter, the Fresnel zone number. For the wave effects to be detectable in a compact-mass gravitational lens, the wavelength $\lambda$ should be comparable with the Schwarzchild radius $R_{\mathrm{s}}$ of the lens [12]. This condition cannot be applied to a string, a non-compact object with conical topology. Instead, we show that the diffraction effects caused by a string are of the leading order with respect to geometrical optics whenever the observation point (either in space or in frequency spectrum) belongs to the low-number Fresnel zone. This is in contrast to the case of a compact-mass lens, for which diffraction scales like $O\left(\lambda / R_{\mathrm{S}}\right)$. Basing on Keller's geometrical theory of diffraction [22], we suggest a simple procedure how the geometrical-optics approximation can be "improved" by adding just two additional paths corresponding to diffraction. These are waves coming from the source to the observer but hitting the string following the shortest path. In this way, the interference effects will be taken into account to the leading order. Our results imply that if a cosmic string exists, it may be identified through a characteristic diffraction pattern in the energy spectrum of the observed signal. Finally, we show that if the string is on the line of sight, the wave amplitude fits the Cornu spiral - the prominent result for the Fresnel diffraction by a straight edge or a slit.

\section{Spacetime of a cosmic string}

We start with a spacetime metric for a static cylindrically symmetric cosmic string $[11,23]$

$$
\mathrm{d} s^{2}=-\mathrm{d} t^{2}+\mathrm{d} r^{2}+(1-4 G \mu)^{2} r^{2} \mathrm{~d} \varphi^{2}+\mathrm{d} z^{2}
$$

where $G$ is the gravitational constant, $\mu$ is the linear mass density of the string lying along the $z$-axis, $(t, r, \varphi, z)$ are cylindrical coordinates, and the system of units in which the speed of light $c=1$ is assumed. With a new angular coordinate $\theta=(1-4 G \mu) \varphi$, the metric (1) takes a Minkowskian form

$$
\mathrm{d} s^{2}=-\mathrm{d} t^{2}+\mathrm{d} r^{2}+r^{2} \mathrm{~d} \theta^{2}+\mathrm{d} z^{2}
$$

which is locally flat, but it globally has a conical topology, since a wedge of angular size $8 \pi G \mu$ is taken out from flat space and the two faces of the wedge are identified $[2,11]$. By introducing the deficit angle $2 \Delta$ with

$$
\Delta=4 \pi G \mu,
$$

the angular coordinate $\theta$ spans the range $2 \pi-2 \Delta$. Solutions of Hamilton's equations [24] for both geometries are depicted in Fig. 1. One can see that geodesics for the metric (1) are curved and deflected an angle $\Delta[11,25]$. However, in coordinates (2) they are just straight lines. Since geodesics passing on opposite sides of the string eventually cross, one should expect interference or diffraction effects. 

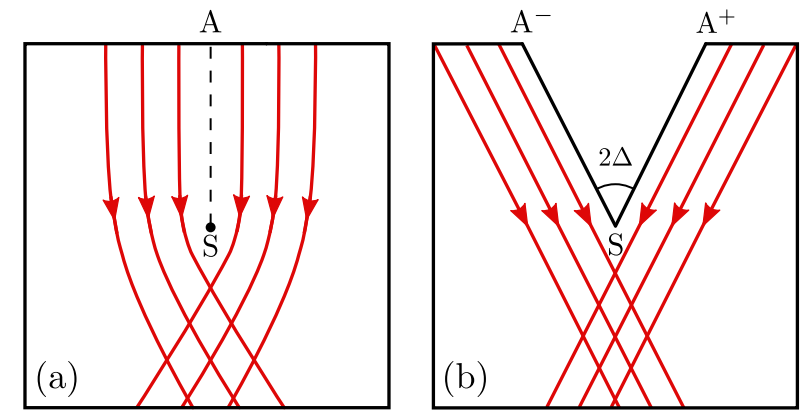

Figure 1: Geodesics in the conical space on $z=0$ plane: (a) curved spacetime; (b) flat spacetime with a deficit angle $2 \Delta$. The cut half-plane $S A$ is perpendicular to the plane of the figure with the edge $S$ coinciding with the string. After the angular transformation the half-plane $S A$ is converted to a wedge of two half-planes $S A^{-}$and $S A^{+}$, which should be identified.

\section{Wave equation in conical space}

We consider the question of finding a solution of the wave equation in background (1) corresponding to a time harmonic distant source, so that the incident waves are plane waves. In order to reduce the problem to two dimensions, the waves are assumed to be emitted in the direction orthogonal to the string. Similarly to Ref. [17], we write the wave equation for a scalar field $U(r, \varphi)$ as

$$
\left(\frac{\partial^{2}}{\partial r^{2}}+\frac{1}{r} \frac{\partial}{\partial r}+\frac{1}{\beta^{2} r^{2}} \frac{\partial^{2}}{\partial \varphi^{2}}+\omega^{2}\right) U=0,
$$

where we denoted $\beta=1-\Delta / \pi$. We assume that Eq. (4) is valid for electromagnetic waves, as well as for gravitational waves when the effect of gravitational lensing on polarization is negligible and both types of waves can be described by a scalar field [26]. A plane wave of unit amplitude incident from the direction $\varphi_{0}$ is described by

$$
U=\mathrm{e}^{\mathrm{i} k r \cos \left\{\beta\left(\varphi-\varphi_{0}\right)\right\}} .
$$

Next, unlike Ref. [17], we perform the coordinate transformation taking advantage of the flat background (2). To do that, we place the cut line $S A$ strictly perpendicular to the wavefront of the incident wave, as shown in Fig. 1(a), so we get $\partial_{\varphi} U=0$ at the cut. Then, we assign the values $\varphi_{0}^{-}=-\pi$ to the left and $\varphi_{0}^{+}=\pi$ to the right of the line $S A$. After angular transformation $\theta=\beta \varphi$, the line $S A$ converts to the wedge $S A^{-}, S A^{+}$given by the angles $\pm(\pi-\Delta)$. The incident field (5) will now be represented by two plane waves

$$
U=\mathrm{e}^{\mathrm{i} k r \cos (\theta \pm \Delta)}
$$

incoming from the directions $\pm(\pi-\Delta)$ with wavefronts perpendicular to the faces of the wedge and propagating in a flat background [see Fig. 1(b)]. As 
we are going to show now, this problem can be reduced to the canonical problem of diffraction on a perfectly conducting half-plane screen solved exactly by Sommerfeld [19-21].

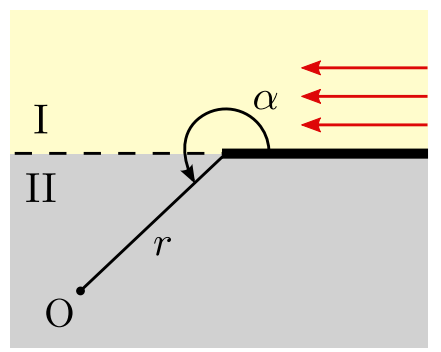

Figure 2: Plane wave grazing a half-plane screen (thick line). The entire space is split into two regions: illuminated (I), shadow (II).

Let us consider a single plane wave grazing an infinite half-plane screen, as shown in Fig. 2. Following Sommerfeld [20], the exact solution for the field at any point $O(r, \alpha)$ can be written in the compact form

$$
U=\mathrm{e}^{-\mathrm{i} k r \cos \alpha} \mathcal{F}\left(\sqrt{2 k r} \cos \frac{\alpha}{2}\right),
$$

where $r$ is the distance from the screen edge, $\alpha$ is the angle measured from the surface of the screen facing the source, and $\mathcal{F}(u)=e^{-\mathrm{i} \pi / 4} \pi^{-1 / 2} \int_{-\infty}^{u} \mathrm{e}^{\mathrm{i} s^{2}} \mathrm{~d} s$ is the Fresnel integral [27]. In Eq. (7) we have taken into account the zero angle of incidence and the Neumann boundary condition on the screen, $\partial_{\alpha} U(r, 0)=0$. It can be verified that solution (7) contains both the geometrical-optics (GO) and the diffracted (D) fields. Indeed, for the angles $0<\alpha<\pi$, in the limit $k r \rightarrow \infty$ far away from the edge, one gets $\mathcal{F} \rightarrow 1$ and $U=\mathrm{e}^{-\mathrm{i} k r \cos \alpha}$, which is the GO incident field. Whereas, for the angles $\pi<\alpha<2 \pi$, one obtains $\mathcal{F} \rightarrow 0$ giving $U=0$ at infinity. The Fresnel function $\mathcal{F}$ smooths the discontinuity of the GO solution across the shadow boundary $\alpha=\pi$ making the total field continuous everywhere. This smooth transition constitutes the diffraction phenomenon [20]. It should also be noted that the original Sommerfeld problem treats two possible boundary conditions on the screen (Dirichlet or Neumann) depending on the polarization of the incident field. However, for grazing incidence, only one polarization can propagate which corresponds to the Neumann condition. On the other hand, the zero field condition is unphysical for the conical space we consider.

Having defined the solution for a single half-plane, we now construct the wave field corresponding to the geometry of Fig. 1(b), in which we have two plane waves (6) grazing the faces of the wedge. Substituting grazing angles: $\alpha=\pi-\Delta \mp \theta$ into Eq. (7), we obtain the total field $U(r, \theta)$ at the observation point $O$

$$
U=\mathrm{e}^{\mathrm{i} k r \cos (\Delta+\theta)} \mathcal{F}\left(w^{+}\right)+\mathrm{e}^{\mathrm{i} k r \cos (\Delta-\theta)} \mathcal{F}\left(w^{-}\right)
$$


with $w^{ \pm}=\sqrt{2 k r} \sin [(\Delta \pm \theta) / 2]$. It describes the wave effects in the gravitational lensing by a cosmic string. It is easy to verify that for $\Delta=0$, it reduces to the unlensed field $U_{0}=\mathrm{e}^{\mathrm{i} k r \cos \theta}$, which is a usual plane wave in Minkowskian space.

For further analysis Eq. (8) can be rewritten in a more convenient form in terms of the eikonals $s^{ \pm}=r \cos (\Delta \pm \theta)$ of the GO waves. The arguments of the Fresnel function become $w^{ \pm}=\sigma^{ \pm} \sqrt{k\left(r-s^{ \pm}\right)}$where $\sigma^{ \pm} \equiv \operatorname{sgn}(\Delta \pm \theta)$ are sign functions giving +1 in the region illuminated by the corresponding GO wave and -1 in the shadow. We get

$$
U=\mathrm{e}^{\mathrm{i} k s^{-}} \mathcal{F}\left(\sigma^{-} \sqrt{\pi N^{-}}\right)+\mathrm{e}^{\mathrm{i} k s^{+}} \mathcal{F}\left(\sigma^{+} \sqrt{\pi N^{+}}\right),
$$

with $N^{ \pm}=k\left(r-s^{ \pm}\right) / \pi$, the number of half-wavelengths matched in the path difference $r-s^{ \pm}$. According to geometrical optics, one can distinguish a doubleimaging region illuminated by both GO waves, $-\Delta<\theta<\Delta$, in which two images are seen at the observation point [see Fig. 3(a)]; and a single-imaging region outside, in which one of the GO waves is shadowed [Fig. 3(b)]. The obtained Eq. (9) gives more complex spatial structure due to diffraction. It determines Fresnel zones which consist of two families of nested parabolas, all with a common focus at the string. In $(r, \theta)$ polar coordinates the parabolas are given by: $r\{1-\cos (\Delta \pm \theta)\}=(\lambda / 2) N_{F}$, with their directrices perpendicular to the lines $\theta=\mp \Delta$, respectively. Each parabola corresponds to a constant phase difference $k\left(r-s^{ \pm}\right)$, and the positive integers $N_{F}$ mark the Fresnel zones numbers [28].
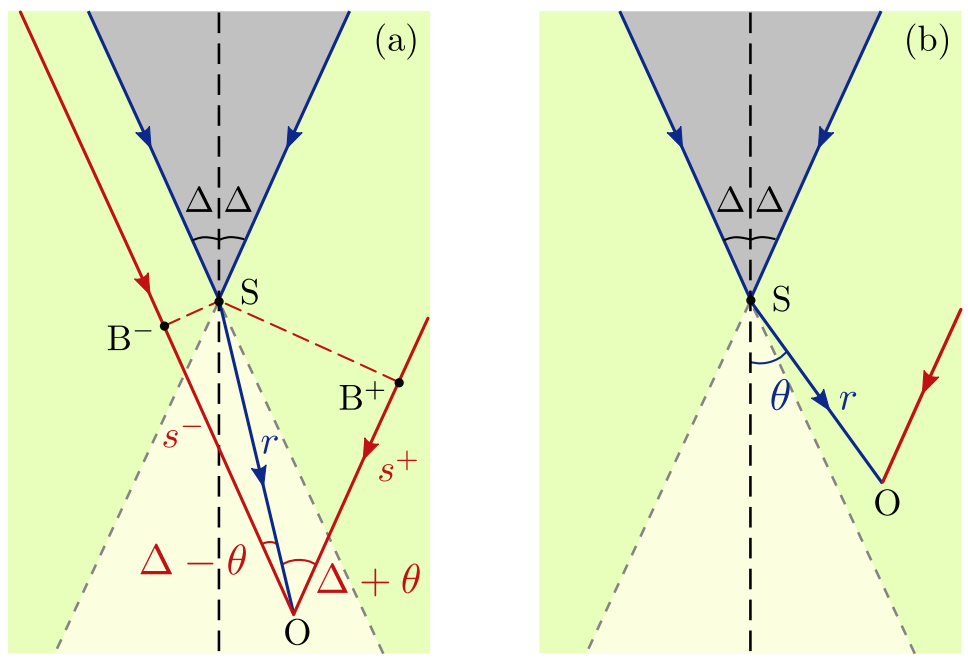

Figure 3: Geometrical theory of diffraction in conical space. The deficit wedge $2 \Delta$ is shadowed. Geometrical-optics (red) and diffraction (blue) rays determine the leading order contribution at the observation point $O$. The distances from $O$ to $B^{ \pm}$are equal to $s^{ \pm}$. 


\section{Geometrical theory of diffraction}

To proceed further, we make asymptotic expansion of the Fresnel integrals for large arguments [20]

$$
\mathcal{F}(w)=\mathcal{H}(w)-\frac{\mathrm{e}^{\mathrm{i} \pi / 4}}{2 \sqrt{\pi}} \frac{\mathrm{e}^{\mathrm{i} w^{2}}}{w}+O\left(w^{-3}\right) .
$$

Here, $\mathcal{H}(w)$ is the Heaviside step function. Substituting in Eq. (9), we obtain

$$
U=h^{-} \mathrm{e}^{\mathrm{i} k s^{-}}+h^{+} \mathrm{e}^{\mathrm{i} k s^{+}}+\left(D^{-}+D^{+}\right) \frac{\mathrm{e}^{\mathrm{i} k r}}{\sqrt{2 \pi k r}} .
$$

The first two terms with notation $h^{ \pm} \equiv \mathcal{H}(\Delta \pm \theta)$ describe the GO waves. The step functions guarantee that the GO waves only contribute to the respective illuminated regions. The third term is the leading order term of the diffracted field. It describes a cylindrical wave emanating from the string vertex and whose amplitude depends on the direction through the "diffraction coefficients":

$$
D^{ \pm}=-\frac{\mathrm{e}^{\mathrm{i} \pi / 4}}{2} \frac{1}{\sin \left[\frac{1}{2}(\Delta \pm \theta)\right]} .
$$

Note that, since the diffracted wave compensates the discontinuity of GO waves, the coefficients $D^{ \pm}$must go to infinity at the corresponding shadow lines $\theta=$ $\pm \Delta$. The solution (11) can thus be interpreted in the framework of Keller's geometrical theory of diffraction [22]. For the double-imaging region, the total wave field at a point $O$ [see Fig. 3(a)] is determined by the sum of: two GO rays coming from the source to the observer directly and two D rays going from the source but hitting the edge - the string location - following the shortest path (Fermat's principle for edge diffraction [22]). In the single-imaging region, only one of the GO rays but both D ones contribute [Fig. 3(b)]. Notice, that D-ray terms in Eq. (11) asymptotically $\sim O\left[\left(N_{F}\right)^{-1 / 2}\right]$ that makes them of the leading order, along with the GO ones, at the points located in the low-number Fresnel zones.

\section{Discussion of the results}

Let us analyze the obtained solution for the case when the string is on the observer-source line of sight $(\theta=0)$. Due to the symmetry, the contributions from both sides are equal, thus the total field normalized to its unlensed value will be of double amplitude as Eq. (9) shows:

$$
\left(\frac{U}{U_{0}}\right)_{\theta=0}=2 \mathrm{e}^{-\mathrm{i} \pi N} \mathcal{F}(\sqrt{\pi N}) .
$$

We observe that finally the wave field on the line of sight is determined by only one parameter

$$
N=\frac{k r}{\pi}(1-\cos \Delta)=\frac{r-s}{\lambda / 2},
$$


which is the number of half-wavelengths matched in the path difference between the GO and D rays. For the modulus of the field we obtain

$$
|U|_{\theta=0}=2|\mathcal{F}(\sqrt{\pi N})| .
$$

The amplification by a factor of 2 is a distinguishing feature of the gravitational lensing by string. It is related to the appearance of double images of identical brightness when the observer is on the line of sight or nearby [11,23]. The field (15) as a function of the dimensionless parameter $N$ is plotted in Fig. 4 . We see the oscillating pattern similar to that for the straight-edge diffraction [20, 29], but with some differences: for the diffraction by string, the shadow part of the curve is missing and the magnitude is doubled. The whole range of values of the field can be visualized by means of the Cornu spiral [Fig. 4(b)]. Here, the
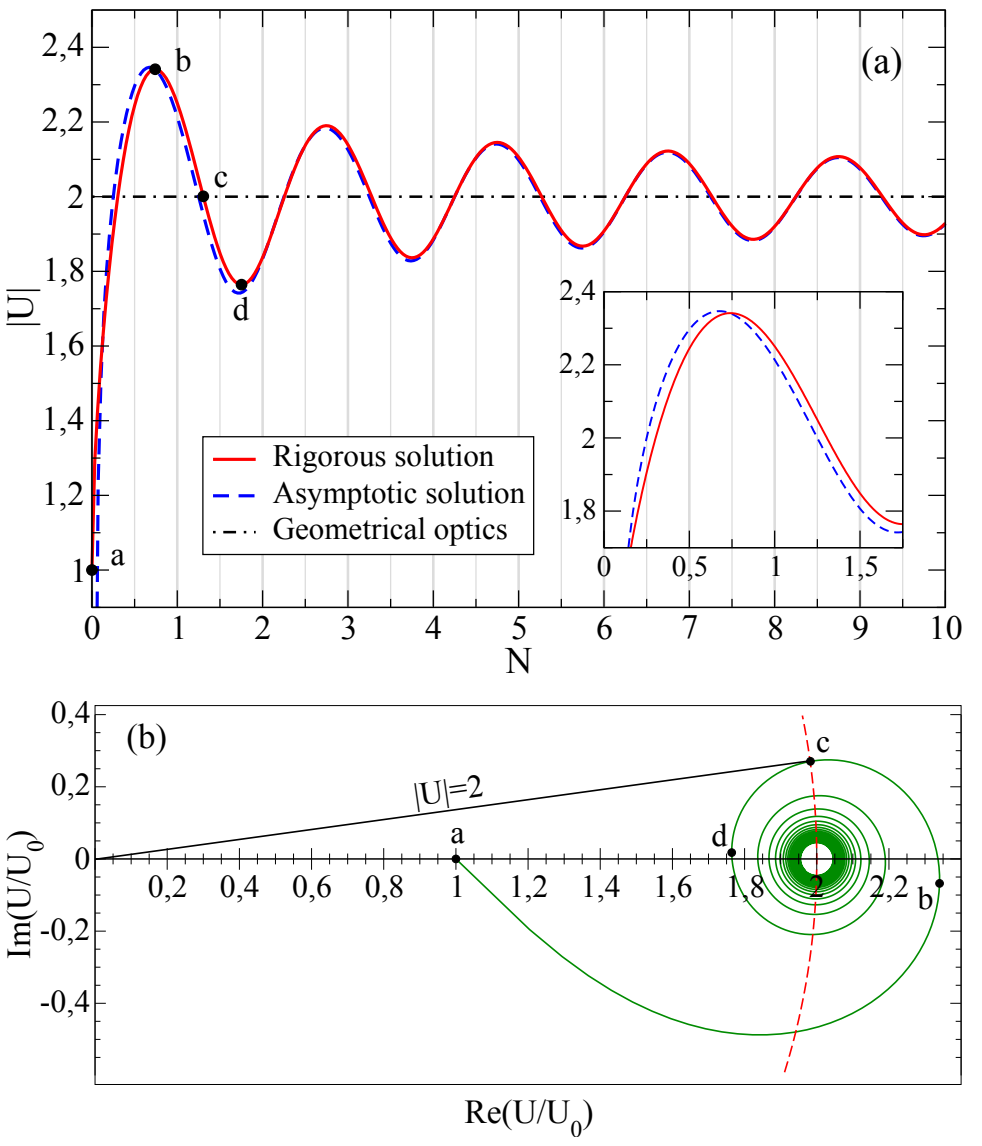

Figure 4: Wave field (a) and Cornu spiral (b) for the case when the string is on the line of sight. The equivalent points on two figures are labeled. The cross points of circular arc (red) with Cornu spiral correspond to $|U|=2$ geometricaloptics values. 
real and imaginary part of the wave field (13) are depicted for different values of $N$. (We omit the phase factor $\mathrm{e}^{-\mathrm{i} \pi N}$ which does not contribute to the absolute value.) In the figure, the length of the cord between the origin and any point of the spiral is $|U|$ and the length of the Cornu curve between the end point $a(N=0)$ and one on the spiral is $\sqrt{\pi N}$. So, the spiral represents a mapping of real $N$-axis on the complex $\mathcal{F}$-plane. As $N \rightarrow \infty$, both the wave field in Fig. 4(a) and the spiral approach the value of 2 , the limiting GO value. Some illustrative points in Fig. 4 have been highlighted. To get more insight into the solution (15), one can use the asymptotic expansion (10). We get

$$
|U|_{\theta=0} \approx 2\left[1-\frac{1}{\pi \sqrt{N}} \cos \left(\pi N+\frac{\pi}{4}\right)\right]^{1 / 2} .
$$

The comparison of the rigorous and asymptotic solutions in Fig. 4(a) indicates a little difference, which for $N \gtrsim 2$ becomes negligible. Therefore, we can use Eq. (16) to determine the maxima and minima of the diffraction:

$$
N=-\frac{1}{4}+n \quad \text { with } \quad \begin{array}{ll}
n=1,3,5 \ldots & \text { maxima } \\
n=2,4,6 \ldots & \text { minima }
\end{array}
$$

The highest maximum occurs at $N_{1} \approx 3 / 4$ giving the field $\left|U_{\max }\right| \approx 2[1+$ $2 /(\pi \sqrt{3})]^{1 / 2} \approx 2.34$ (point $b$ in Fig. 4). This value corresponds to the intensity $I_{\max }=\left|U_{\max }\right|^{2} \approx 5.47$. The diffraction pattern given by Fig. 4 can, in principle, be detected in two different ways: (i) if the observation frequency is fixed, one can interpret the variation of field with $N$ as variation with distance $r$ between the string and the observer, since $N \sim r$. In this case, the first maximum will be located at a distance

$$
r_{1}=N_{1} \frac{\lambda}{2} \frac{1}{1-\cos \Delta} \approx N_{1} \frac{\lambda}{\Delta^{2}},
$$

where the last approximation corresponds to $\Delta \ll 1$. (ii) On the other hand, if $r$ is fixed, one could detect the field oscillations caused by diffraction in the frequency spectrum due to $N \sim k \sim \omega$. In this case, the maximum amplification will be detected at $\lambda^{*} \approx r \Delta^{2} / N_{1}$. It should also be noted that the asymptotic solution (16), which we have obtained from the Fresnel integral, in the limit $\Delta \ll 1$ coincides with the one obtained by Suyama et al. [17] by different approach.

When the string is not on the line of sight, $\theta \neq 0$, the wave paths from two images are different, $s^{+} \neq s^{-}$, therefore one should expect the interference effects (constructive or destructive) even for the GO waves (for $\theta=0$ it is always constructive). If we write out the GO field from Eq. (11)

$$
\left|U_{\mathrm{GO}}\right|=\left[h^{+}+h^{-}+2 h^{+} h^{-} \cos (2 k r \sin \theta \sin \Delta)\right]^{1 / 2},
$$

we obtain the wave oscillations amplified by a factor of 2 in the double-imaging region, and neither interference nor amplification $\left(\left|U_{\mathrm{GO}}\right|=1\right)$ in the singleimaging region. However, if the full solution is considered, which includes the 
diffraction terms, the behavior becomes qualitatively different. This is illustrated in Fig. 5 where the total solution (9) and the GO field (19) are shown as functions of the angle $\theta$. For convenience, we normalize $\theta$ by $\Delta$, so that the

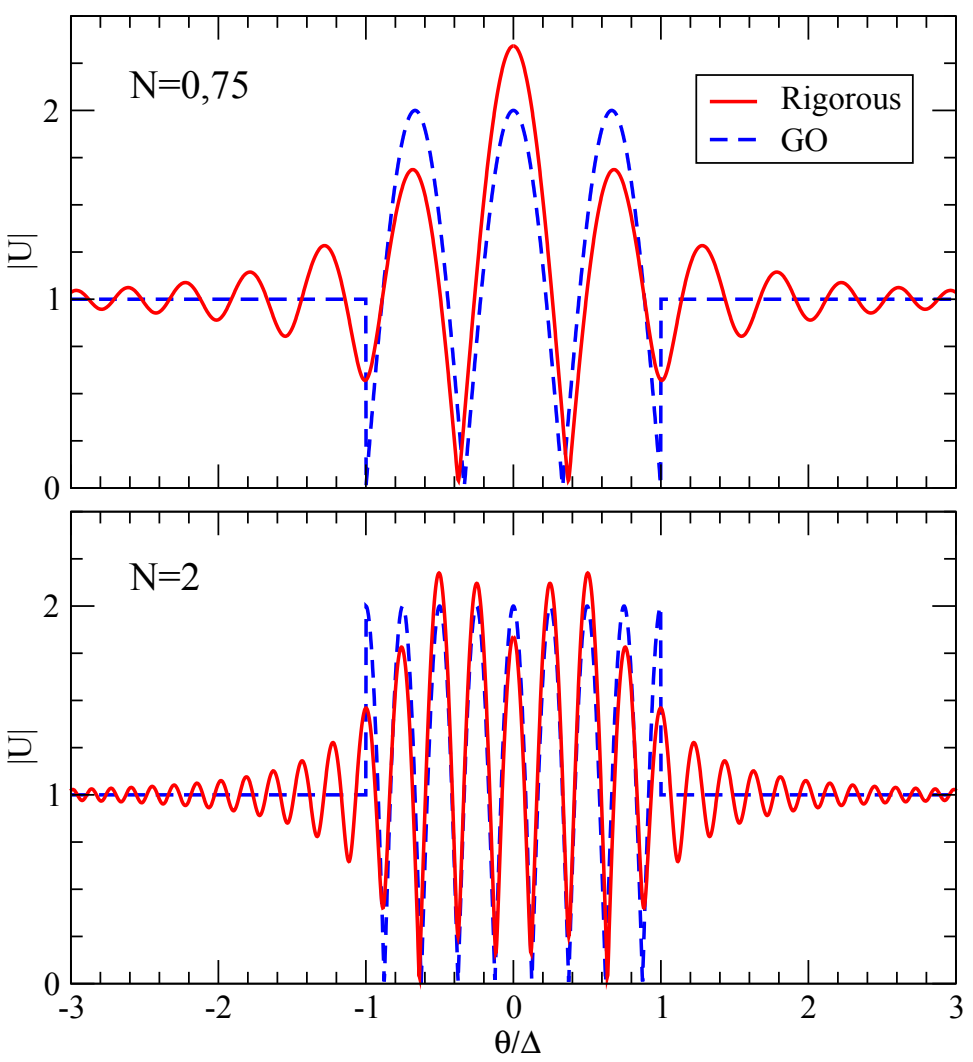

Figure 5: Comparison of the rigorous and geometrical optics solution of the field $|U|$ in terms of the observation angle $\theta$ normalized to $\Delta$, for $N=0.75$ and $N=2$ with $\Delta=0.01 \pi$.

boundary between the double and single-imaging regions corresponds to \pm 1 . We see that diffraction adds new features to the interference pattern: (i) a higher than 2 amplification in wave magnitude; (ii) a smooth transition in the amplitude shape when crossing the shadow boundaries; (iii) an amplification and oscillations in the single-imaging region. The oscillation spacing in the central part of the pattern can be estimated approximately from the GO field. From Eq. (19) we find

$$
\sin \theta_{m}=\frac{1-\cos \Delta}{\sin \Delta} \frac{m}{2 N} \quad \text { with } m \in \mathbb{Z},
$$

where even $m$ determines the maxima and odd $m$, the minima. In the limit $\Delta \ll 1$ which implies $\theta \ll 1$ for the double-imaging region, this relation is 
simplified to

$$
\frac{\theta_{m}}{\Delta}=\frac{m}{4 N}
$$

where the $m$ values are limited by $-4 N \leq m \leq 4 N$. Thus, the angular spacing between two consecutive maxima or minima will be $\delta \theta \approx \Delta /(2 N)$.

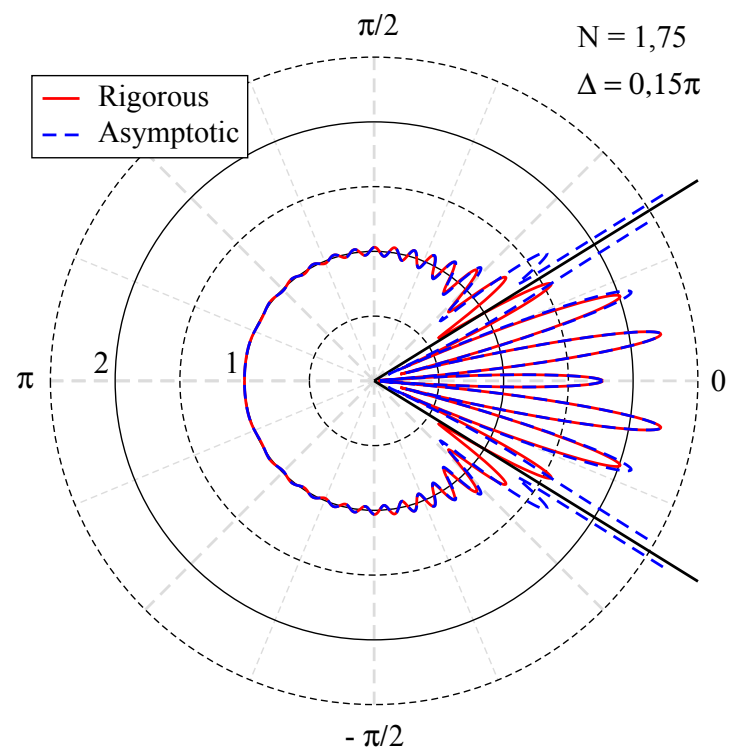

Figure 6: Angular dependence of wave field $|U|$ caused by plane wave incident from $\varphi=\pi$ direction in conical space (1), as compared with asymptotic solution. The field values correspond to fixed $k r$ determined by $N=1.75$ and $\Delta=0.15 \pi$. The double-imaging region is bounded by two black radial lines.

Note that all the results obtained for the angle $\theta$ in flat space can be expressed in terms of the "physical" angle $\varphi$ in curved space by means of the substitution $\theta=\beta \varphi$. To get more insight into the angular distribution of the field in $\varphi$-space spanning the range of $2 \pi$, we present in Fig. 6 a polar plot for the field $|U|$ given by exact solution (9) along with its asymptotic limit (11). For a better view, a rather large value of the deficit angle $\Delta$ is taken. It is seen that both solutions almost coincide for all directions except at the shadow lines $\varphi= \pm \Delta / \beta$, where the diffraction coefficients (12) diverge. This singularity, however, can be overcome in the framework of so-called "uniform theory of edge diffraction" [30], which makes the asymptotic solution to be finite and continuous everywhere including at the shadow boundaries.

\section{Conclusions and perspectives}

In conclusion, the analytical theory we have presented in this Letter, describes propagation of scalar waves in conical spacetime created by a straight 
cosmic string. Even though the string scenario for galaxy formation requires a small deficit angle, $\Delta \ll 1[2]$, we believe that our results are also applicable for condensed matter systems for which a wider range of $\Delta$ is observed. For instance, the effective geometry (1) describes propagation of sound near a topological defect (disclination) in nematic liquid crystals, where $c$ is the velocity of sound [31]. As long as the D field decays to 0 as it approaches the boundary $\pm \pi$ (see Fig. 6), our assumptions in the model are justified. It would also be interesting to treat this problem as the diffraction by a cone, or a wedge, given the geometry of the string, using different boundary conditions and another setting $[32,33]$.

The solutions obtained for an infinitely distant source are shown to be determined by just one parameter, that is the Fresnel zone number. In particular, it indicates, for a given string tension, the typical wavelength at which one should expect the oscillations in the spectrum due to diffraction. Taking the typical value $\Delta \sim 10^{-7}$ and a distance to the string within our galaxy, $r \sim 10^{20} \mathrm{~m}$, one would obtain the highest amplification at $\nu \approx 200 \mathrm{~Hz}$, which is in the frequency band of Laser Interferometer Gravitational-wave Observatory (LIGO). The derivation can proceed in a similar way when the source is located at a finite distance. In this case, the parameter $N$ will include dependence on the sourcestring distance and the Fresnel-zone structure will be determined by nested hyperbolas [28].

Basing on geometrical theory of diffraction, we suggest an intrinsically simple method that allows to explain diffraction phenomena in spacetime generated by a cosmic string or similar topological defect within the principles of geometrical optics. Namely, the wave field at an observation point is determined to the leading order by interference of a few characteristic rays: the geometric-optics and the diffracted ones. This method, we believe, may be easily applied to other geometries.

\section{Acknowledgements}

IFN acknowledges financial support from Universitat de Barcelona under the APIF scholarship.

\section{References}

\section{References}

[1] T. W. B. Kibble, J. Phys. A 9, 1387 (1976).

[2] A. Vilenkin and E. P. S. Shellard, Cosmic Strings and Other Topological Defects (Cambridge University Press, Cambridge, 1994).

[3] M. B. Hindmarsh and T. W. B. Kibble, Rep. Prog. Phys. 58, 477 (1995).

[4] G. A. Williams, Phys. Rev. Lett. 82, 1201 (1999). 
[5] E. Witten, Phys. Lett. B 153, 243 (1985).

[6] M. J. Bowick, L. Chandar, E. A. Schiff, and A. M. Srivastava, Science 263, 943 (1994).

[7] M. A. H. Vozmediano, M. I. Katsnelson, and F. Guinea, Physics Reports, 496, 109 (2010).

[8] T. G. Mackay and A. Lakhtakia, Phys. Lett. A 374, 2305 (2010).

[9] T.H. Anderson, T. G. Mackay, and A. Lakhtakia, Phys. Lett. A 374, 4637 (2010).

[10] I. I. Smolyaninov, V. N. Smolyaninova, and A. I. Smolyaninov, Phil. Trans. R. Soc. A 373, 20140360 (2015).

[11] A. Vilenkin, Phys. Rev. D 23, 852 (1981).

[12] S. Deguchi and W. D. Watson, Phys. Rev. D 34, 1708 (1986).

[13] P. Schneider, J. Ehlers, and E. Falco, Gravitational Lenses (Springer, New York, 1992).

[14] B. Linet, Ann. Inst. Henri Poincare A 45, 249 (1986).

[15] D. L. Osipov, JETP Lett. 62, 765 (1995).

[16] K. Yamamoto and K. Tsunoda, Phys. Rev. D 68, 041302 (2003).

[17] T. Suyama, T. Tanaka, and R. Takahashi, Phys. Rev. D 73, 024026 (2006).

[18] C.-M. Yoo, R. Saito, Y. Sendouda, K. Takahashi, and D. Yamauchi, Prog. Theor. Exp. Phys. 013E01 (2013).

[19] A. Sommerfeld, Mathem. Ann. 47, 317 (1896).

[20] A. Sommerfeld, Lectures on Theoretical Physics, Vol. IV, Optics (Academic Press, New York, 1954).

[21] A. Sommerfeld, Mathematical Theory of Diffraction (Birkhäuser, Boston, 2004).

[22] J. B. Keller, J. Opt. Soc. Am. 52, 116 (1962).

[23] J. R. Gott, Astrophys. J. 288, 422 (1985).

[24] I. Fernández-Núñez and O. Bulashenko, Phys. Lett. A 380, 1 (2016).

[25] A. Vilenkin, Astrophys. J. 282 L51 (1984).

[26] C. W. Misner and K. S. Thorne, and J. A. Wheeler, Gravitation (W. H. Freeman and Company, San Francisco, 1973). 
[27] L. D. Landau and E. M. Lifshitz, Electrodynamics of Continuous Media 2nd ed., (Pergamon, New York, 1984).

[28] I. Fernández-Núñez and O. Bulashenko, (unpublished results).

[29] M. Born and E. Wolf, Principles of Optics, 7th ed. (Cambridge University Press, Cambridge, 1999).

[30] S. W. Lee and G. A. Deschamps, IEEE Trans. Antennas Propag. 24, 25 (1976).

[31] E. Pereira, S. Fumeron, and F. Moraes, Phys. Rev. E 87, 022506 (2013).

[32] J. S. Dowker, J. Phys. A 10, 115 (1977).

[33] S. A. Fulling, C. S. Trendafilova, P. N. Truong, and J. Wagner, J. Phys. A 45, 374018 (2012). 